\title{
Canine Intestinal Organoids in a Dual-Chamber Permeable Support System
}

\author{
Vojtech Gabriel ${ }^{1}$, Christopher Zdyrski ${ }^{1}$, Dipak K. Sahoo ${ }^{2}$, Kimberly Dao ${ }^{3}$, Agnes Bourgois-Mochel ${ }^{2}$, Todd Atherly ${ }^{3}$, Marilyn \\ N. Martinez ${ }^{4}$, Donna A. Volpe ${ }^{5}$, Jamie Kopper ${ }^{2}$, Karin Allenspach ${ }^{2,3}$, Jonathan P. Mochel ${ }^{1,3}$ \\ ${ }^{1}$ Department of Biomedical Sciences, College of Veterinary Medicine, lowa State University ${ }^{2}$ Department of Veterinary Clinical Sciences, College of \\ Veterinary Medicine, lowa State University ${ }^{3}$ DD Health Solutions Inc. ${ }^{4}$ Office of New Animal Drug Evaluation, Center for Veterinary Medicine, Food and \\ Drug Administration ${ }^{5}$ Division of Applied Regulatory Science, Office of Clinical Pharmacology, Office of Translational Sciences, Center for Drug Evaluation \\ and Research, Food and Drug Administration
}

\section{Corresponding Authors}

Vojtech Gabriel

vojt.gabriel@gmail.com

Karin Allenspach

allek@iastate.edu

Jonathan P. Mochel

jmochel@iastate.edu

\section{Citation}

Gabriel, V., Zdyrski, C., Sahoo, D.K., Dao, K., Bourgois-Mochel, A.,

Atherly, T., Martinez, M.N.,

Volpe, D.A., Kopper, J., Allenspach, K.,

Mochel, J.P. Canine Intestinal Organoids in a Dual-Chamber Permeable Support

System. J. Vis. Exp. (181), e63612,

doi:10.3791/63612 (2022).

\section{Date Published}

March 2, 2022

\section{DOI}

$10.3791 / 63612$

\section{URL}

jove.com/video/63612

\section{Abstract}

The permeable support system is typically used in conjunction with traditional twodimensional (2D) cell lines as an in vitro tool for evaluating the oral permeability of new therapeutic drug candidates. However, the use of these conventional cell lines has limitations, such as altered expression of tight junctions, partial cell differentiation, and the absence of key nuclear receptors. Despite these shortcomings, the Caco-2 and MDCK models are widely accepted and validated for the prediction of human in vivo oral permeability.

Dogs are a relevant translational model for biomedical research due to their similarities in gastrointestinal anatomy and intestinal microflora with humans. Accordingly, and in support of parallel drug development, the elaboration of an efficient and accurate in vitro tool for predicting in vivo drug permeability characteristics both in dogs and humans is highly desirable. Such a tool could be the canine intestinal organoid system, characterized by three-dimensional (3D), self-assembled epithelial structures derived from adult stem cells.

The (1) Permeable Support Seeding Protocol describes the experimental methods for dissociating and seeding canine organoids in the inserts. Canine organoid isolation, culture, and harvest have been previously described in a separate set of protocols in this special issue. Methods for general upkeep of the canine intestinal organoid monolayer are discussed thoroughly in the (2) Monolayer Maintenance Protocol. Additionally, this protocol describes methods to assess the structural integrity of the monolayer via transepithelial electrical resistance (TEER) measurements and light microscopy. Finally, the (3) Permeability Experimental Protocol describes the tasks directly preceding an experiment, including in vitro validation of experimental results. 
Overall, the canine organoid model, combined with a dual-chamber cell culture technology, overcomes limitations associated with 2D experimental models, thereby improving the reliability of predictions of the apparent oral permeability of therapeutic drug candidates both in the canine and human patient.

\section{Introduction}

Permeable support systems are typically used to determine the apparent permeability of therapeutic drug candidates through the intestinal epithelial barrier ${ }^{1,2}$. They can also be employed to assess cellular secretion ${ }^{3}$, cell migration ${ }^{4}$, and drug toxicity ${ }^{5}$. In vitro oral drug permeability assays are a key step in the drug discovery and development process $^{2}$, with individual drug candidates being tested at the early stage of the drug R\&D lifecycle ${ }^{6}$. The permeable support system is a dual-chamber cell culture apparatus consisting of an insert with a semiporous membrane placed in a multiwell plate. This system allows direct access to the apical and basolateral sides of a cell-monolayer grown in the insert ${ }^{7}$. The monolayer used in these systems is typically derived from gastrointestinal epithelial cells (e.g., human colorectal adenocarcinoma Caco-2 cell line $)^{8}$. Cell cultures grow in a polarized state mimicking the natural microarchitecture of intestinal epithelial cells, enabling further cellular differentiation, similar microanatomy and function ${ }^{7}$. Details of the permeable support insert can be found in Figure 1. The seeding of the inserts with $2 \mathrm{D}$ cell cultures, traditionally used for assessing intestinal drug permeability, is relatively affordable and easy to culture ${ }^{9}$. These systems present several major limitations, including their limited capacity to predict the intestinal metabolism of therapeutic drug candidates ${ }^{10,11}$. This is true for all mechanisms of drug absorption, be it passive absorption through the tight junctions in-between epithelial cells, active transepithelial absorption through efflux, or uptake transporters (e.g., P- glycoprotein, monocarboxylate transporter 1), and drugs that are metabolized by enterocytes.

Dogs share a common environment and diet with humans ${ }^{12}$. Canine intestinal anatomy and microbiome composition closely resemble that of humans ${ }^{13}$, which has been attributed to domestication and shared diets over the past 36,000 years ${ }^{14}$. Unfortunately, these similarities can also be common causes/triggers for disease development. Dogs develop similar chronic morbidities to humans, such as obesity ${ }^{15}$, inflammatory bowel disease ${ }^{16}$, colorectal adenocarcinoma ${ }^{17}$, gastrointestinal stromal tumor $(\mathrm{GIST})^{18}$, and various other pathologies associated with their relative longevity ${ }^{19}$. Accordingly, canine organoids may be successfully used for reverse translational research of these chronic multifactorial diseases in the spirit of the One Health Initiative ${ }^{20}$.

Caco-2 cells are the most used cell lines for drug oral absorption assays ${ }^{21}$. These cells are currently considered the "gold standard" model for in vitro intestinal permeability assays ${ }^{2,22,23}$. The Caco-2 cell line expresses efflux and uptake transporters found in the human intestinal tract, although at different expression levels ${ }^{24,25,26}$. Caco-2 cells are also widely used as models to determine if a drug is a substrate or inhibitor of intestinal efflux transporters 22,27 . Although the Caco-2 cells are of colonic origin, they mimic an enterocyte cell. Unfortunately, Caco-2 cells only represent one cell type from the epithelial layer of the small 
intestine ${ }^{9}$, which fails to recapitulate the complex intestinal epithelial cell type composition accurately. For example, goblet cells dedicated to mucus production are absent from Caco-2 cultures such that mucus-drug interactions cannot be assessed without coculture with other cell lines ${ }^{28}$. Furthermore, Caco-2 cultures do not express several of the important nuclear receptors typically present in the intestine, such as pregnane $X$ receptor (PXR), steroid $X$ receptor (SXR), and constitutive androstane receptor $(\mathrm{CAR})^{29}$. Consequently, Caco-2 cultures fail to model the induction of drug transporters and enzymes by certain drugs that are inducers of these receptors (e.g., rifampin) ${ }^{30}$.

The 3D intestinal organoid technology addresses some of these limitations $^{19}$. Organoids are self-assembled constructs derived from adult stem cells that can be established from tissue samples harvested using microinvasive techniques ${ }^{20}$. Human-induced pluripotent stem cells are being employed for intestinal permeability models ${ }^{31,32}$. Canine organoids provide a relevant alternative to human organoids because human stem cell research is restricted by ethical issues ${ }^{33}$. Furthermore, canine organoids provide an in vitro system for exploring canine drug permeability, metabolism, active transport, and drug-drug interactions. To address this technology gap, the consistent and reliable growth of canine intestinal organoids in a permeable support system has been described ${ }^{34}$. A permeability assay with canine intestinal organoids may potentially predict canine intestinal permeability and metabolism of small drug molecules compared to currently used assays (Caco-2). Confirmation of these pivotal features lends this novel in vitro system to future work exploring the potential impact of inducers on intracellular metabolism and active transport.
Canine organoids are composed of all the cell types typically present in the epithelial layer of the intestine. From a functional and microanatomical view, they reliably replicate the environment of the epithelial layer of the canine gut ${ }^{19,35}$. Furthermore, the presence of mucus, canine-specific drug transporters and enzymes, and overall cellular differentiation in canine intestinal organoids is comparable to what is seen in vivo in dogs ${ }^{34}$. Thus, organoids can be isolated from diseased veterinary patients and used to model the effect of various disease processes (e.g., chronic intestinal inflammation) on canine oral drug permeability ${ }^{19,36}$. The canine intestinal organoid system can also be used in other settings than drug permeability experiments. These 3D structures can also be isolated from diseased patients as previously described by Chandra et al. for inflammatory bowel disease, colorectal adenocarcinoma, and gastrointestinal stromal tumor $^{19}$.

The Permeable Support Seeding Protocol describes methods for establishing canine intestinal organoid cultures in the inserts. This first protocol outlines methods to dissociate established canine organoid cultures plated in the extracellular membrane matrix. Furthermore, the precoating of the inserts with collagen I and the extracellular membrane matrix is discussed in this protocol. Embedding canine organoids in the permeable support inserts is also described in detail.

The second protocol is the Monolayer Maintenance Protocol, which includes general upkeep of canine 3D organoids plated in an insert. The frequency and volumes of the organoid media used to refresh the culture, and ways to prevent cell culture damage, are presented in this second protocol, along with experimental methods for assessing the confluency of the epithelial monolayer. 
Finally, the Permeability Experimental Protocol focuses on ways to determine if the canine intestinal $3 D$ organoids in a permeability assay are ready for experimental use and the verification steps needed before conducting any experiment. This section also describes the setup and the successful execution of a permeability experiment, along with the incubation and sampling of therapeutic drug candidates in the chambers of the monolayer culture. The use of the low permeability fluorescein isothiocyanate (FITC-dextran) to monitor monolayer integrity is also discussed. Finally, an in vitro evaluation method for validating the results after the conclusion of an experiment is described. Permeability experiments are an extremely vast topic and are very well summarized by Hubatsch et al. ${ }^{37}$. The workflow of the protocols is summarized in Figure 2.

\section{Canine Intestinal Organoids on a Permeable Support}

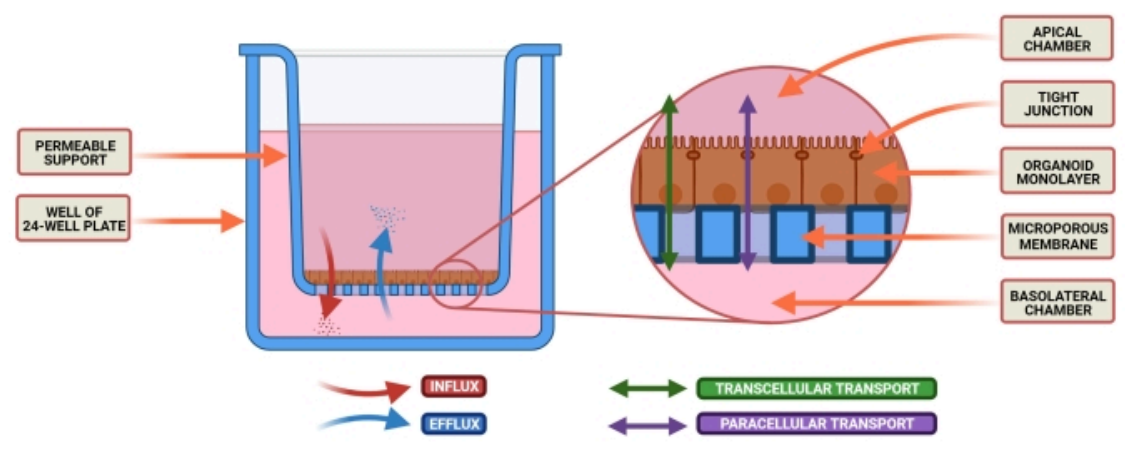

Figure 1: Canine intestinal organoids on a permeable support system. The permeable support insert is positioned in a well of a 24-well plate. The microporous membrane allows for the seeding of dissociated canine intestinal organoids, and these cells will eventually form an organoid 2D monolayer. This technology allows access to both the AP and BL sides of the monolayer. Organoid medium is introduced in both the AP and BL chambers of the permeable support. The absorption $(\mathrm{AP} \rightarrow \mathrm{BL})$ and secretion $(\mathrm{BL} \rightarrow \mathrm{AP})$ of the drug candidate are illustrated, as well as two possible modes of drug transport.

Abbreviations: $\mathrm{AP}=$ apical; $\mathrm{BL}=$ basolateral. Please click here to view a larger version of this figure. 


\section{Workflow of Canine Organoid Permeable Support Protocols}

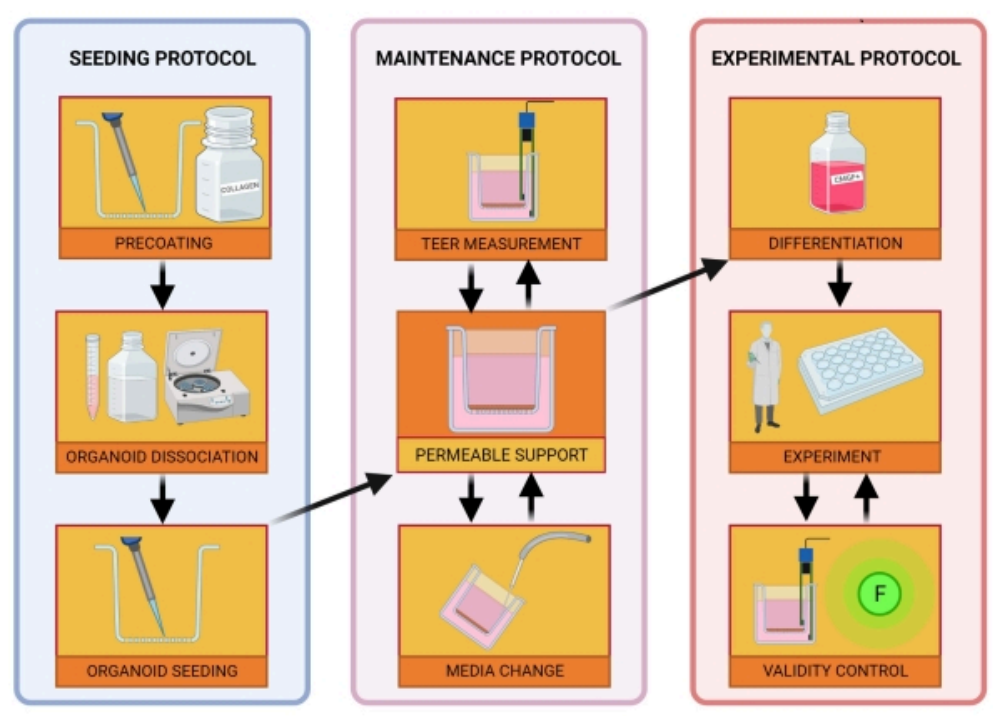

Figure 2: Workflow of canine organoid permeable support protocols. The permeable support insert is precoated with a mixture of the extracellular membrane matrix and collagen I and incubated for $1 \mathrm{~h}$. During the incubation process, the organoid culture is dissociated. Individual organoid cells are seeded in the insert, medium in the basolateral chamber is added immediately after seeding, while medium to the apical chamber is added $24 \mathrm{~h}$ after the seeding process concludes. Maintenance and monitoring of the organoids include regular medium changes, TEER value measurements, and light microscopy to evaluate the integrity of the monolayer. Before the experiment, the organoids must be differentiated by removing ROCK inhibitor and GSKi $\beta$ from the media. The TEER values are measured on the day of the experiment, and the organoid monolayer is inspected via light microscopy for damage to the cells. Medium is then exchanged for an appropriate buffer and incubated prior to the experiment. The FITC-dextran assay is used during intestinal permeability experiments ${ }^{39}$ as a marker of monolayer integrity. TEER measurements are taken after the experiment, and light microscopy will validate the results after $24 \mathrm{~h}$. Abbreviations: TEER = transepithelial electrical resistance; ROCK = rho-associated kinase; GSKi $\beta=$ glycogen synthase kinase beta; $F=$ fluorescence. Please click here to view a larger version of this figure.

\section{Protocol}

The research was approved and performed in compliance with the Institutional Animal Care and Use Committee of lowa State University (IACUC-19-337; IACUC-18-065; IACUC-19-017). The following section (steps 1.1-1.3) describes the Permeable Support Seeding Protocol, and the procedures are summarized in Figure 3. 


\section{Workflow of the Permeable Support Seeding Protocol}

PRECOATING OF PERMEABLE SUPPORTS

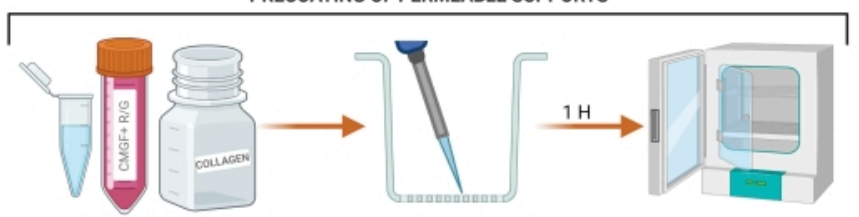

MASTER MIX

INSERT PRECOATING

INCUBATION
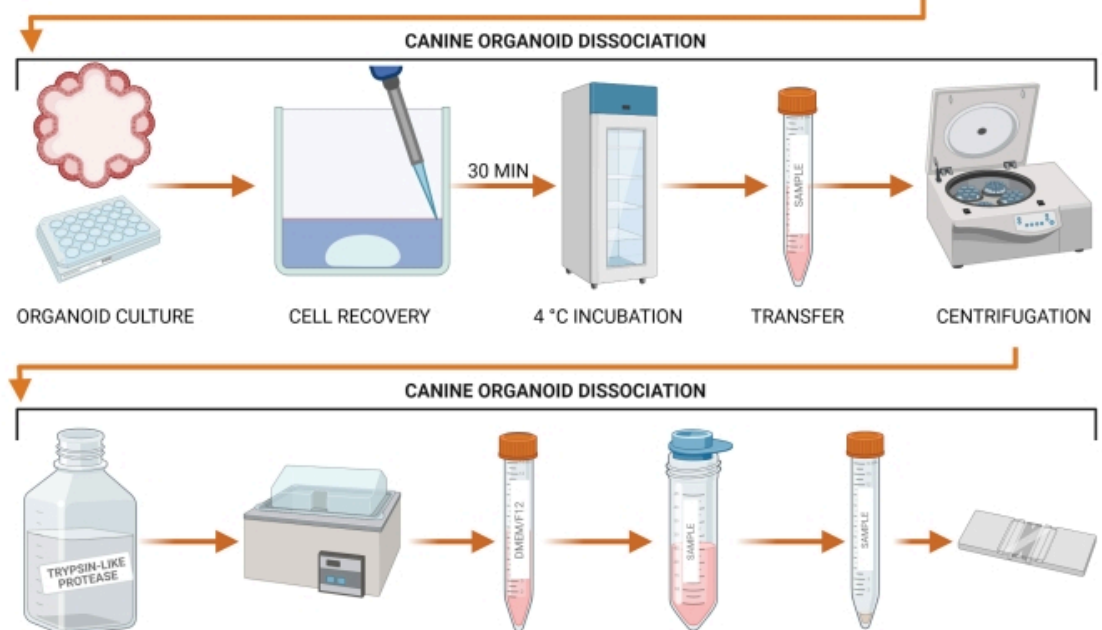

DISSOCIATION $\quad 37^{\circ} \mathrm{C}$ INCUBATION DMEM/F12 WASH STRAINING PELLET CELL COUNTING

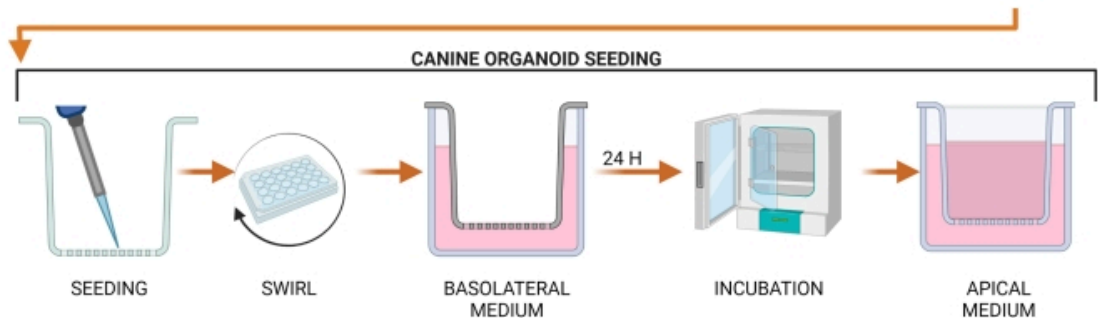

Figure 3: Workflow of the permeable support seeding protocol. The permeable support inserts are precoated with a combination of CMGF+ R/G, collagen I, and the extracellular membrane matrix and subsequently incubated. Media from the canine organoid culture is aspirated and replaced with a Cell Recovery Solution, followed by a 30 min incubation at $4{ }^{\circ} \mathrm{C}$. The culture is subsequently transferred to a tube, and organoid dissociation is performed using trypsin-like protease. Undissociated organoids are removed by passage through a strainer to achieve a single-cell suspension, and cell concentration is determined using a hemocytometer or an automated cell counter. The cells are seeded on a permeable support insert, and CMGF+ R/G is added to the basolateral chamber. The culture is then incubated for $24 \mathrm{~h}$, and the remaining liquid is removed from the apical chamber and replaced with CMGF+R/G. Abbreviations: ROCK = rho-associated kinase; GSKi $\beta$ = glycogen synthase kinase beta; CMGF + R/G = Complete medium with growth factors enhanced with ROCK inhibitor and GSKiß. Please click here to view a larger version of this figure. 


\section{Permeable support seeding protocol}

1. Precoating of the permeable support inserts

1. Prepare Complete medium with growth factors enhanced with Rho-associated protein kinase (ROCK) inhibitor and glycogen synthase kinasebeta inhibitor (GSKiß) $(\mathrm{CMGF}+\mathrm{R} / \mathrm{G})$ according to the information in Table 1.

2. Prepare an ice bucket and begin thawing the extracellular membrane matrix on ice. Place a 24well plate containing the needed number of inserts in the incubator to prewarm. Collect rat tail collagen I $(3 \mathrm{mg} / \mathrm{mL})$ and place it on ice while protecting from light. Collect $\mathrm{CMGF}+\mathrm{R} / \mathrm{G}$ and place it on ice.

3. Calculate the total number of inserts and blanks required for the experiment, keeping aside $100 \mu \mathrm{L}$ of the coating solution for each insert.

NOTE: Prepare more coating solution than needed; preparing at least $15 \%$ more than needed is recommended.

4. In a $15 \mathrm{~mL}$ tube, mix CMGF+ R/G with the extracellular membrane matrix (1\%) and collagen I $(1 \%)$ and gently pipet mix.

5. Coat each polyester insert with $100 \mu \mathrm{L}$ of the coating solution and place the inserts in the incubator $\left(37^{\circ} \mathrm{C}\right.$; $5 \% \mathrm{CO}_{2}$ atmosphere) for $1 \mathrm{~h}$.

6. After the incubation, carefully aspirate the coating solution off each insert using a vacuum aspirator or a P1000 pipette, being careful not to disturb the insert filter. Place a precoated plate in the incubator to keep warm.

2. Canine organoid dissociation
NOTE: Use canine organoids that have been cultured for at least four days. Before beginning dissociation, refer to Gabriel et al. ${ }^{38}$ to determine when a sample is healthy, dense, and sufficient for experimentation. It is recommended to dissociate one extra well of organoids for every well plating procedure. Furthermore, increasing the desired number of inserts by $\sim 20 \%$ to account for uneven organoid growth or damage caused by improper manipulation is recommended. If planning to use FITCdextran, prepare extra wells.

1. Prepare an ice bucket and a vial of cold $1 \mathrm{x}$ Advanced DMEM/F12 stock in the biosafety cabinet.

2. Place the extracellular membrane matrix on ice to begin thawing, submerging it in ice to protect against rapid thawing and avoid solidification. Place a box of pipette tips (P200) in the freezer for the plating of the extracellular membrane matrix.

3. Prechill a refrigerated centrifuge to $4{ }^{\circ} \mathrm{C}$.

4. Move $\mathrm{CMGF}+\mathrm{R} / \mathrm{G}$ from the freezer/refrigerator to a $37^{\circ} \mathrm{C}$ water bath. Avoid direct light exposure when possible.

5. Remove all medium from 24 -well plate with organoid culture for an appropriate number of wells ( 1 well of a 24-well plate per 2-4 inserts) while taking care to not disturb the extracellular membrane matrix.

NOTE: The volume might vary depending on the cell counting system used.

6. Add $0.5 \mathrm{~mL}$ of prechilled Cell Recovery Solution per well to dissolve the extracellular membrane matrix domes.

7. Incubate the plate in the refrigerator $\left(4^{\circ} \mathrm{C}\right)$ for 30 $\min$. 
8. Pipette the suspension, collect all organoids and dissolved extracellular membrane matrix, and transfer them to a $15 \mathrm{~mL}$ tube.

9. Centrifuge $\left(700 \times g\right.$ for 5 min at $\left.4{ }^{\circ} \mathrm{C}\right)$ and remove the supernatant until the level reaches the $0.5 \mathrm{~mL}$ mark, making sure not to disturb the pellet.

10. Add $1 \mathrm{~mL}$ of the trypsin-like protease and incubate in the $37^{\circ} \mathrm{C}$ water bath for $8 \mathrm{~min}$. Flick the tube several times during the incubation period to mix the cells.

11. Transfer the tube with the sample back to a biosafety cabinet and slowly add $7 \mathrm{~mL}$ of prechilled Advanced $\mathrm{DMEM} / \mathrm{F} 12$ to inactivate the trypsin-like protease and stop cell dissociation.

12. Prewet a $40 \mu \mathrm{m}$ cell strainer with $1 \mathrm{~mL}$ of Advanced DMEM/F12. Gently pipet the mixture and filter the suspension through; pipette additional Advanced DMEM/F12 to rinse the strainer.

13. Centrifuge the tube $\left(700 \times g\right.$ for $5 \min$ at $\left.4{ }^{\circ} \mathrm{C}\right)$ and remove the supernatant. Do not disturb the pellet.

14. Resuspend the cell pellet in $\sim 50-100 \mu \mathrm{L}$ of culture medium $(\mathrm{CMGF}+\mathrm{R} / \mathrm{G})$ for every well of organoids that was disassociated.

15. Count a subsample of the suspension $(\sim 10 \mu \mathrm{L})$ using a hemocytometer or appropriate machine and determine the total cell number in the suspension.
3. Canine organoid seeding

1. Dilute or concentrate the cell suspension to obtain a cell concentration of $\sim 75,000$ cells per $\mathrm{mL}$.

2. Seed $100 \mu \mathrm{L}$ of the suspension into each insert using BSA (1\%) precoated tips to avoid cell adhesion during transfer. Add one coated insert as a cellfree blank without any organoids growing while still receiving regular media changes.

3. Gently swirl the plate in a circular motion for $\sim 30 \mathrm{~s}$ to disperse the seeded cells across the insert. Confirm via light microscopy the even distribution of cells.

4. Add $700 \mu \mathrm{L}$ of $\mathrm{CMGF}+\mathrm{R} / \mathrm{G}$ to the basolateral chamber and place the plate in the incubator $\left(37^{\circ} \mathrm{C}\right.$; $5 \% \mathrm{CO}_{2}$ atmosphere) for $24 \mathrm{~h}$.

5. After $24 \mathrm{~h}$, gently remove the cell suspension from the apical chamber and replace it with $200 \mu \mathrm{L}$ of $\mathrm{CMGF}+\mathrm{R} / \mathrm{G}$. Return the plate to the incubator.

\section{Organoid cell monolayer maintenance protocol}

NOTE: The following section (steps 2.1-2.2) describes the organoid cell monolayer maintenance protocol. The workflow of procedures presented in this protocol is summarized in Figure 4. A table for note-taking that can help with the standardization of TEER value measurements is presented in

\section{Supplemental Table 1}


Workflow of the Permeable Support Culture Maintenance

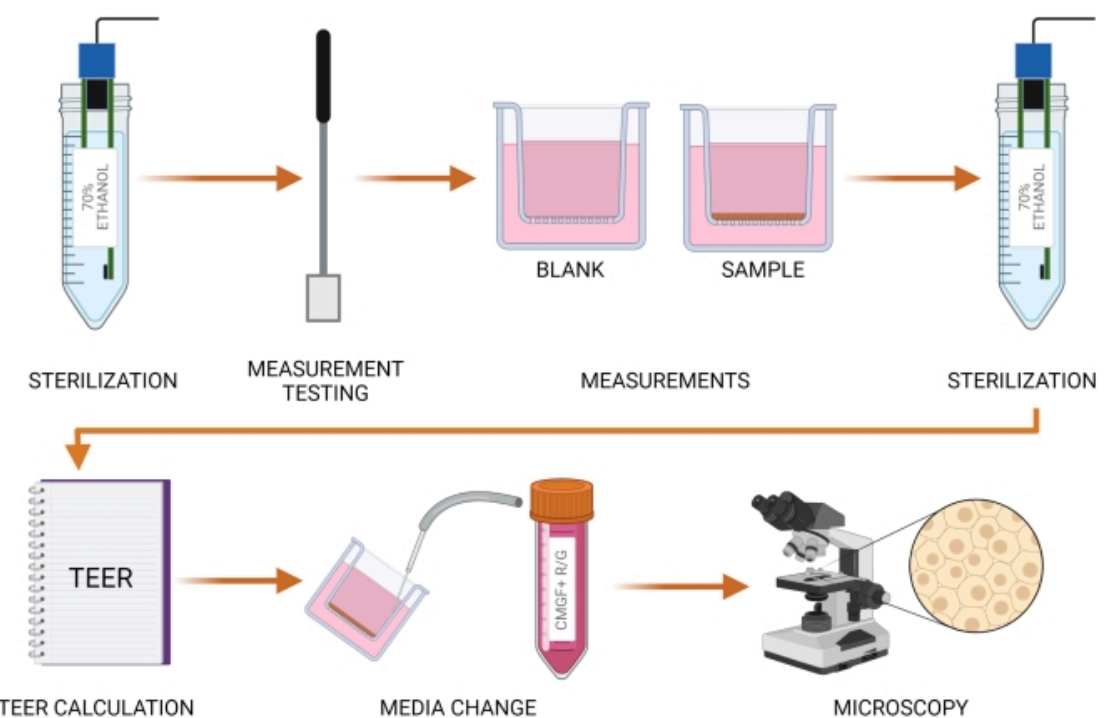

Figure 4: Workflow of the permeable support culture maintenance. TEER values are measured using electrodes (probes) and a volt/ohm meter. Probes must be chemically sterilized with $70 \%$ alcohol prior to inserting into the wells. The blank and organoid cell inserts are measured, and TEER values are calculated. Medium is subsequently refreshed in both apical and basolateral chambers, and the canine organoid culture on the insert is visualized using light microscopy. Tears in either organoid culture or microporous membrane are noted and handled according to the protocol. Abbreviation: TEER = transepithelial electrical resistance. Please click here to view a larger version of this figure.

1. TEER value measurement

NOTE: TEER value measurements are performed using an Epithelial Volt/Ohm Meter with chopstick attachment. See the manufacturer's instructions for use. TEER values provide information on the integrity of the canine organoid monolayer.

1. Take TEER value measurements on every alternative day during cell culture growth.

2. Move the Epithelial Volt/Ohm Meter and its electrodes to the biosafety cabinet. Chemically sterilize the electrodes in $70 \%$ alcohol before use.
Set the function to Ohms. Wait at least one minute until the electrodes dry.

3. Before taking the first measurement, insert the wire electrode into the port and turn on the power. Ensure that the meter displays $1,000 \Omega$ with the wire electrode insert instead of measuring chopsticks. If this is not the case, adjust the device.

4. Insert the electrodes in the apical and basolateral chamber of the cell-free insert (blank), so the apical chamber contains the shorter electrode, and the basolateral chamber contains the longer electrode 
(as seen in Figure 4). Do not touch the membrane, but at the same time, ensure that the electrodes are submerged in the medium.

5. Wait for a few seconds until the value stabilizes and note the value in a lab book. Measure the remaining canine organoid monolayers, making sure to sterilize the electrodes with $70 \%$ alcohol when measuring different samples. Take care not to touch the organoid monolayer with the electrode.

6. After measurements are taken, sterilize the electrodes with $70 \%$ alcohol for the last time. Be sure to protect them from damage caused by inappropriate manipulation and store them according to the manufacturer's instructions.

7. Calculate the TEER values for every well using

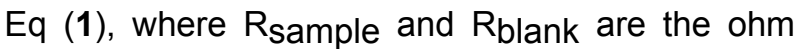
$(\Omega)$ values from the monolayer and blank wells, respectively, and the area $\left(\mathrm{cm}^{2}\right)$ is that of the insert. $\operatorname{TEER}\left(\Omega \times \mathrm{cm}^{2}\right)=\left(R_{\text {Sample }}-R_{\text {Blank }}\right) \times$ Area $\left[\mathrm{cm}^{2}\right]$

2. Monolayer upkeep

NOTE: Review the recommended medium change plan in Table 2.

1. Using sterile disposable 9" Pasteur pipets and a vacuum aspirator, gently aspirate the medium from the apical and then the basolateral chambers. Tilt the plate to see the medium surface clearly. Avoid aspirating too close to the microporous membrane in the apical chamber to prevent damage to the cell monolayer.

NOTE: Use a new Pasteur pipet when moving between samples. Pipettes are also a possible replacement for this procedure.
2. Slowly add $\mathrm{CMGF}+\mathrm{R} / \mathrm{G}$ using $\mathrm{P} 1000$ pipettes aiming for a wall of the apical or basolateral chamber. Perform the medium change in the apical chamber very carefully to avoid damaging the monolayer.

3. Check the wells every other day under a light microscope, evaluating the health of the culture and monitoring for tears in the organoid monolayer or the microporous membrane. Use phase-contrast microscopy to highlight details of the culture.

NOTE: In the case of canine organoid monolayer tears, the monolayer is given time to recover and regrow. In the case of microporous membrane tears, the well must be excluded from the experiment.

Table 2: Media change recommendation for organoid cultures. $C M G F+R / G$ is changed in the apical and basolateral chamber of the wells every other day on every alternative day. The longer culture period over the weekend demands an increased volume of medium in both basolateral and apical chambers, which is applied on a Friday afternoon and changed on Monday. Abbreviations: ROCK = rhoassociated kinase; GSKi $\beta$ = glycogen synthase kinase beta; $\mathrm{CMGF}+\mathrm{R} / \mathrm{G}=$ Complete medium with growth factors enhanced with ROCK inhibitor and GSKiß. Please click here to download this Table.

\section{Permeability experimental protocol}

NOTE: The following section (steps 3.1-3.5) describes the Permeability Experimental Protocol. The experimental protocol workflow to measure a drug's in vitro permeability is summarized in Figure 5. 
Workflow of the Experimental Protocol
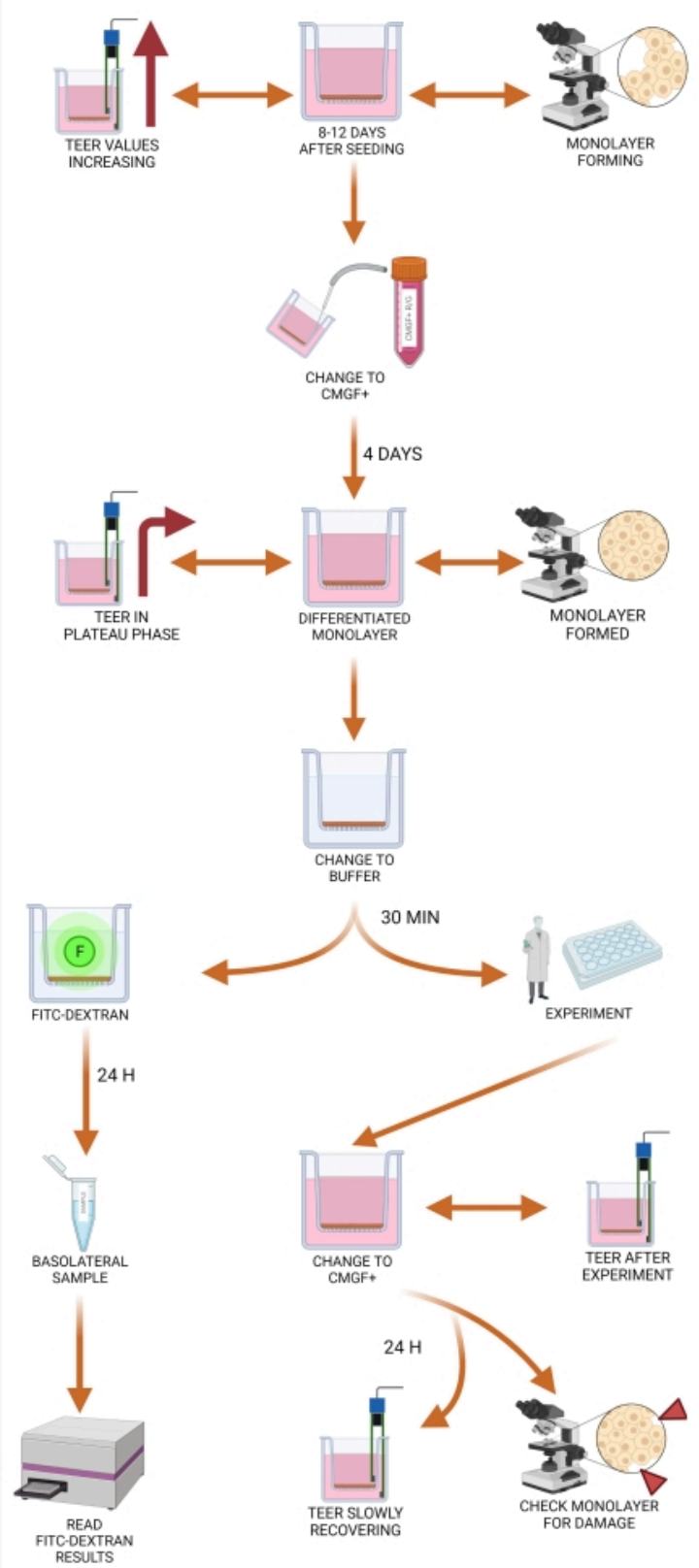

Figure 5: Workflow of the experimental protocol. Organoid medium must be changed from CMGF+ R/G to CMGF + between eight to twelve days after seeding the inserts, allowing for cellular differentiation. After changing the medium (both apical and basolateral) to CMGF+, the TEER values should still be increasing, with the canine organoid monolayer almost reaching confluency. At least four days after the medium is changed to CMGF+, the readiness of the monolayers is evaluated. When the monolayer is completely formed, and TEER values reach a plateau phase (typically between 1,500 and $2,500 \Omega \cdot \mathrm{cm}^{2}$ ), the medium is exchanged for the transport buffer for $30 \mathrm{~min}$ to allow the monolayer to adjust to the new 
environment. At time 0 min of the experiment, the FITC-dextran assay is performed, and the 20 min basolateral sample is collected. Results are subsequently analyzed on a plate reader. After the experiment, the apical and basolateral chamber contents are again changed to CMGF+, and TEER value readings are taken. The monolayers are incubated for $24 \mathrm{~h}$, and the integrity of the monolayer is assessed through repeated TEER measurements. Abbreviations: TEER $=$ transepithelial electrical resistance; ROCK = rho-associated kinase; GSKi $\beta=$ glycogen synthase kinase beta; $C M G F+R / G=$ Complete medium with growth factors enhanced with ROCK inhibitor and GSKi $\beta$; CMGF+ = Complete medium with growth factors but without ROCK inhibitor or GSKiß; FITC = fluorescein isothiocyanate; F = fluorescence. Please click here to view a larger version of this figure.

1. Evaluating organoid monolayer readiness

NOTE: This step occurs 8-14 days after seeding.

1. Check the monolayer at least every other day under the light microscope (use phase-contrast to visualize the monolayer's integrity). Continue to the next step when the cell monolayer is fully formed without gaps or apparent signs of tears.

2. Change the organoid medium from $C M G F+R / G$ to CMGF+ (excluding ROCK inhibitor and GSKi $\beta$ from the medium composition). Swapping the medium at least four days prior to the experiment is recommended.

NOTE: This step will allow for proper differentiation of the organoid monolayer.

3. Continue measuring TEER values approximately every other day. When TEER values start to plateau at approximately 1,500 to $2,000 \Omega \times \mathrm{cm}^{2}$ (Figure 6), measure the TEER values every day.

NOTE: This steady state can be maintained for approximately 2-3 days, which is the optimal window of time to perform permeability testing (usually at days 11 to 13). The plateau TEER values may slightly oscillate based on the intestinal localization of the organoids, measurement temperature, breed, age, and disease state of the dog.
4. Schedule the drug permeability assay immediately to avoid a rapid decrease in TEER values or overgrowth of the organoid monolayer to multiple cell layers.

2. Preparing for the experiment

NOTE: Incubator shakers can be used during the experiment to avoid the effects of the unstirred water layer. Measure TEER values at consistent temperatures.

1. On the day of the experiment, measure TEER values and confirm that the values reached a steady state and are not declining rapidly.

2. Choose the best monolayers (via light microscopy and TEER values) from the excess of $20 \%$ inserts to perform the experiment.

3. Observe the monolayers under a light microscope and exclude incomplete, torn, or overgrown organoid monolayers.

4. Prepare the transport buffer and adjust its $\mathrm{pH}$ to desired values.

NOTE: The composition of the experimental buffer differs based on the experimental setup. A frequently used buffer is composed of Hank's Balanced Salt Solution (HBSS), glucose (12.5 mM), and 4-(2-hydroxyethyl)-1-piperazineethanesulfonic acid 
(HEPES, $25 \mathrm{mM}$ ). This composition ensures the viability of organoid culture during an experiment.

5. Carefully aspirate the medium from the apical and basolateral chambers of the selected wells.

6. Add $200 \mu \mathrm{L}$ of transport buffer to the apical chamber and $800 \mu \mathrm{L}$ to the basolateral chamber.

NOTE: Transport buffer should be first added to the apical chamber and then to the basolateral chamber to avoid detachment of the monolayer from the microporous membrane.

7. Place the plate in the incubator $\left(37^{\circ} \mathrm{C} ; 5 \% \mathrm{CO}_{2}\right.$ atmosphere) for 30 min to equilibrate.

NOTE: The canine organoid monolayers are now ready for the drug permeability experiment.

3. Typical experimental layout-IgY concentration solution NOTE: The experimental design and layout may change depending on the research question. The permeability of immunoglobulin $\mathrm{Y}(\mathrm{Ig} \mathrm{Y})$ through an organoid monolayer is used in the protocol as an example and can be modified. The term donor chamber refers to the chamber where the drug is initially applied, while the receiving chamber refers to the chamber accepting the drug from the donor chamber. A typical experiment collects samples in the receiver chambers over $2 \mathrm{~h}$ (e.g., 15, 30, $60,90,120 \mathrm{~min})$.

1. Prepare the $\lg Y$ solution (drug or solute of choice) by dissolving it in the transport buffer to the desired final concentration. Prepare more drug solution than needed.

NOTE: Drugs with low aqueous solubility may be first dissolved in an organic solvent (e.g., ethanol, DMSO) before adding to the buffer. The final concentration of the solvent should be less than $1 \%$ so as not to damage the cell monolayer.

2. Remove the buffer from the donor chamber (either apical or basolateral chamber) of each well.

3. Add the $\lg Y$ solution (drug solution) to all the donor chambers. Use the remaining solution as the time 0 donor solution for the measurement of the initial drug concentration.

4. At the required time points, remove $50 \mu \mathrm{L}$ from the receiving chamber and place it in a labeled tube. At the last time point, remove a sample from the donor chamber. At the end of the experiment, transfer the donor and receiver aliquots to $\mathrm{a}-20^{\circ} \mathrm{C}$ freezer.

NOTE: If many time points are needed, replacement of buffer in the receiving chamber may be done but must be accounted for in the calculation of the apparent permeability. The concentration in the receiver chamber should not reach more than $10 \%$ of the donor chamber at the end of the experiment to maintain sink conditions ${ }^{44}$.

4. Organoid cell monolayer quality control

NOTE: FITC-dextran solution may be used to confirm monolayer integrity during the experiment. FITC-dextran is used as an example of a monolayer integrity assay. Others include Lucifer yellow, PEG-4000, radiolabeled mannitol, and inulin ${ }^{44}$.

1. At time $0 \mathrm{~min}$, aspirate the contents of the apical chamber and replace with $250 \mu \mathrm{L}$ of FITC-dextran solution $(5 \mathrm{mg} / \mathrm{mL}, 4 \mathrm{kDa})$ in triplicate for each experimental group.

NOTE: Do not expose FITC-dextran to light.

2. After $20 \mathrm{~min}$, remove the buffer from the basolateral chamber. 
3. Measure the fluorescence intensity of the basolateral sample using a fluorescence plate reader (use a calibration curve; excitation set at 485 $\mathrm{nm}$ and emission value at $528 \mathrm{~nm}$ ).

NOTE: Papp of FITC can also be calculated using the method described in the discussion.

4. After the experiment concludes, carefully aspirate excess buffer from the apical and basolateral chambers.

5. Add $200 \mu \mathrm{L}$ of CMGF+ in the apical chamber and $700 \mu \mathrm{L}$ to the basolateral chamber.

6. Measure TEER values in the individual wells

7. Place the plate in the incubator $\left(37^{\circ} \mathrm{C} ; 5 \% \mathrm{CO}_{2}\right.$ atmosphere) for $24 \mathrm{~h}$.

8. If applicable, after $24 \mathrm{~h}$, measure TEER values to assess possible damage to the monolayer during the quality control portion of the experiment. Use light microscopy to visualize the integrity of the canine organoid monolayer.

5. Fixing cell monolayers for downstream analysis

1. Prepare Formalin-Acetic Acid-Alcohol solution (FAA, composition in Table 1).

2. Remove the transport buffer or CMGF+ from the apical and basolateral chambers using a P1000 pipet or sterile disposable 9" Pasteur pipets and a vacuum aspirator.

3. Fill the apical and basal chambers with FAA.

4. After $24 \mathrm{~h}$, aspirate the FAA and replace it with $70 \%$ ethanol.

5. Wrap the plate with flexible laboratory film to prevent evaporation and proceed to block preparation. 


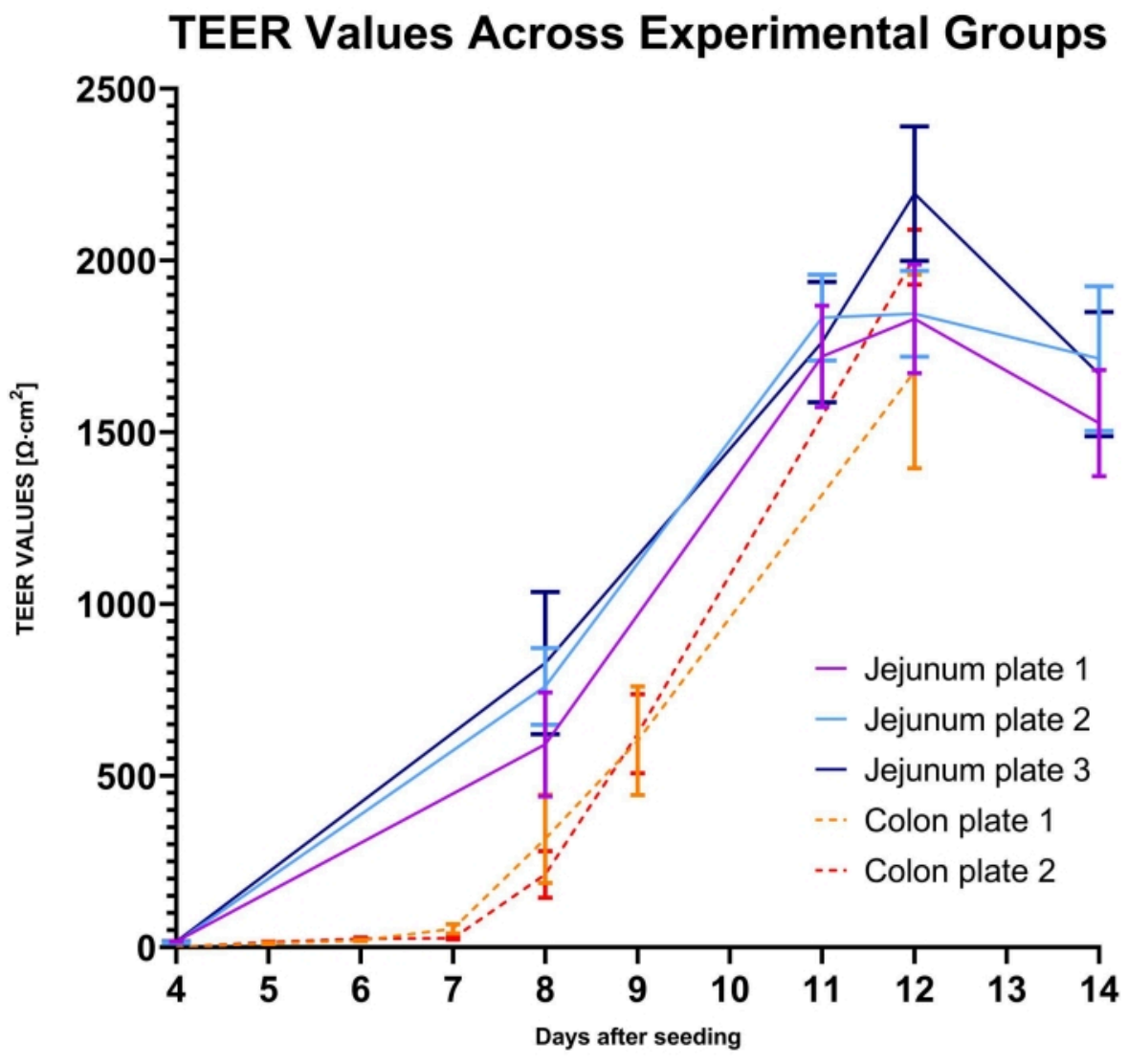

Figure 6: TEER values across experimental groups. TEER values were measured in five groups of canine intestinal organoid monolayers. Three groups were composed of canine jejunal enteroids, and two groups consisted of colonoids. Each group included 12 to 22 replicates. The TEER values for jejunal enteroid cultures are shown from Day 4 to Day 14 , and colonoid cultures are shown from Day 4 to Day 12 (measurements ended as the organoid monolayer reached steadystate values, indicating the monolayer was ready for experimental use). Error bars express SEM of the measurements. Abbreviation: TEER = transepithelial electrical resistance. Please click here to view a larger version of this figure.

\section{Representative Results}

Standard operating procedures for the culture of canine organoids were previously described ${ }^{33}$, and the Canine Organoid Protocol has also been discussed in detail in this special issue ${ }^{38}$. Canine intestinal organoids cultured on permeable supports were fixed and paraffin-embedded to examine the microanatomy of the monolayer and the cell populations. The paraffin-embedding process was previously discussed by Gabriel et al. ${ }^{38}$. Routine staining (hematoxylin and eosin) has been performed, and the Alcian Blue staining technique was used to detect goblet cells in the canine organoid monolayer (see Figure 7). 


\section{Canine Intestinal Organoid Monolayer Staining}

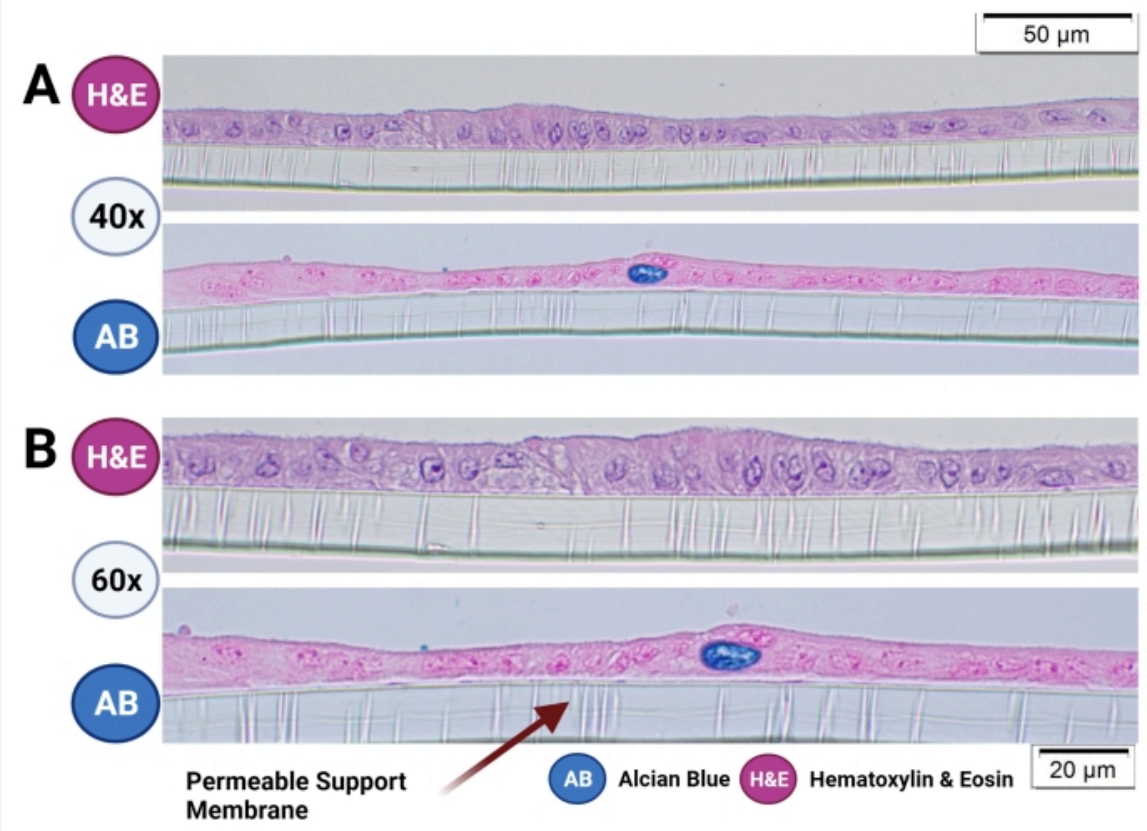

Figure 7: Canine intestinal organoid monolayer staining. Canine intestinal organoids of colonic origin in the permeable support have been paraffin-embedded and stained with $H \& E$ and $A B$. Representative images were taken using light microscopy at 40x (A) and 60x (B) magnification. H\&E staining reveals a columnar epithelial monolayer, and the microvilli in the apical part of the cells can be observed at $60 x$ magnification. $A B$ staining further unveils the presence of goblet cells (dark blue) in the canine intestinal organoid monolayer. Scale bars $=20 \mu \mathrm{m}(\mathbf{A}), 50 \mu \mathrm{m}(\mathbf{B})$. Abbreviations: H\&E $=$ hematoxylin and eosin; $A B=$ Alcian Blue. Please click here to view a larger version of this figure.

The organoid culture on the permeable support should be grown for 10 to 14 days to be ready for a permeability experiment. Light microscopy is used in conjunction with TEER value measurements to confirm the readiness of the organoid culture for permeability testing of drug candidates. Representative light microscopic images of different canine intestinal organoid monolayers from healthy and diseased animals are presented in Figure 8. 
Canine Intestinal Organoid Monolayer Microscopy

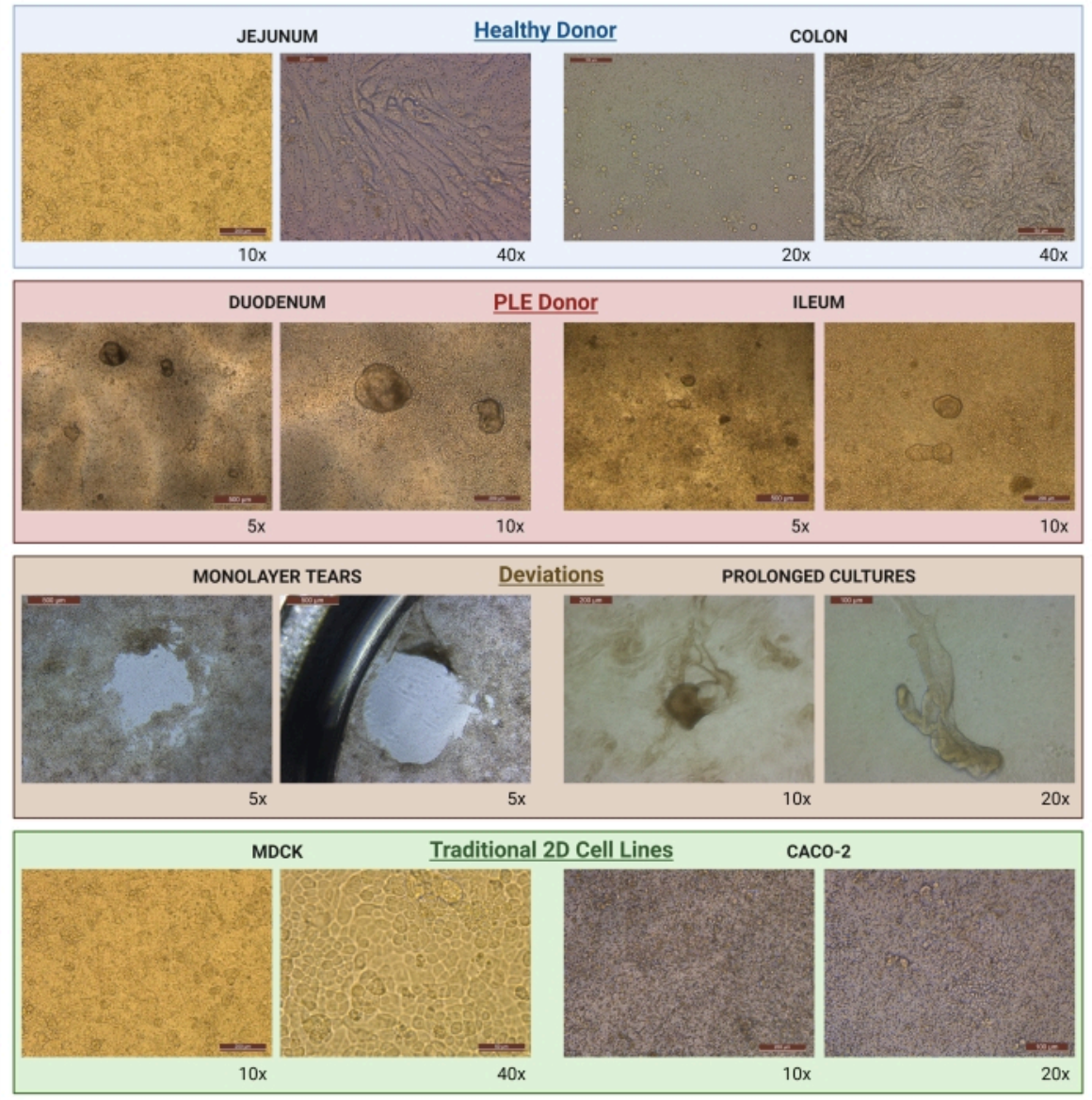

Figure 8: Canine intestinal organoid monolayer microscopy. Images of growing monolayers as seen under light microscopy. Organoid monolayers derived from healthy donors are represented by jejunal enteroid (10x; 40x) and colonoid (20x; 40x) cultures. Organoid monolayers of diseased donors are represented by duodenal (5x; 10x) and ileal (5x; 10x) enteroids derived from a canine patient diagnosed with PLE. Both PLE organoid cultures are examples of improper cell isolation during the seeding of organoids, and these cultures could not be used for drug permeability assays because of the presence of 3D organoids. Examples of deviations from proper monolayer formation, such as monolayer tears $(5 \mathrm{x})$, are caused by uncareful manipulation of the biological samples. The prolonged culture of organoids (10x; 20x) is caused by the long upkeep of the organoids on the insert when the organoids begin to resemble their original 3D structure. For comparison, images of traditional 2D cell lines on the permeable supports used commonly for drug permeability studies are presented (MDCK-10x; 40x, and Caco-2-10x; 20x). Abbreviations: PLE = protein-losing enteropathy; MDCK = MadinDarby canine kidney. Scale bars $=500 \mu \mathrm{m}(\mathbf{5 x}), 200 \mu \mathrm{m}(\mathbf{1 0 x}), 100 \mu \mathrm{m}(\mathbf{2 0 x}), 50 \mu \mathrm{m}(\mathbf{4 0 x})$. Please click here to view a larger version of this figure. 
Organoid monolayers were also fixed in $3 \%$ Paraformaldehyde-3\% Glutaraldehyde in phosphate-buffered saline (PBS). Transmission electron microscopy (TEM) was used to characterize the ultrastructure of the organoid culture on the permeable supports. Microanatomical structures of microvilli and tight junctions can be seen in Figure 9.

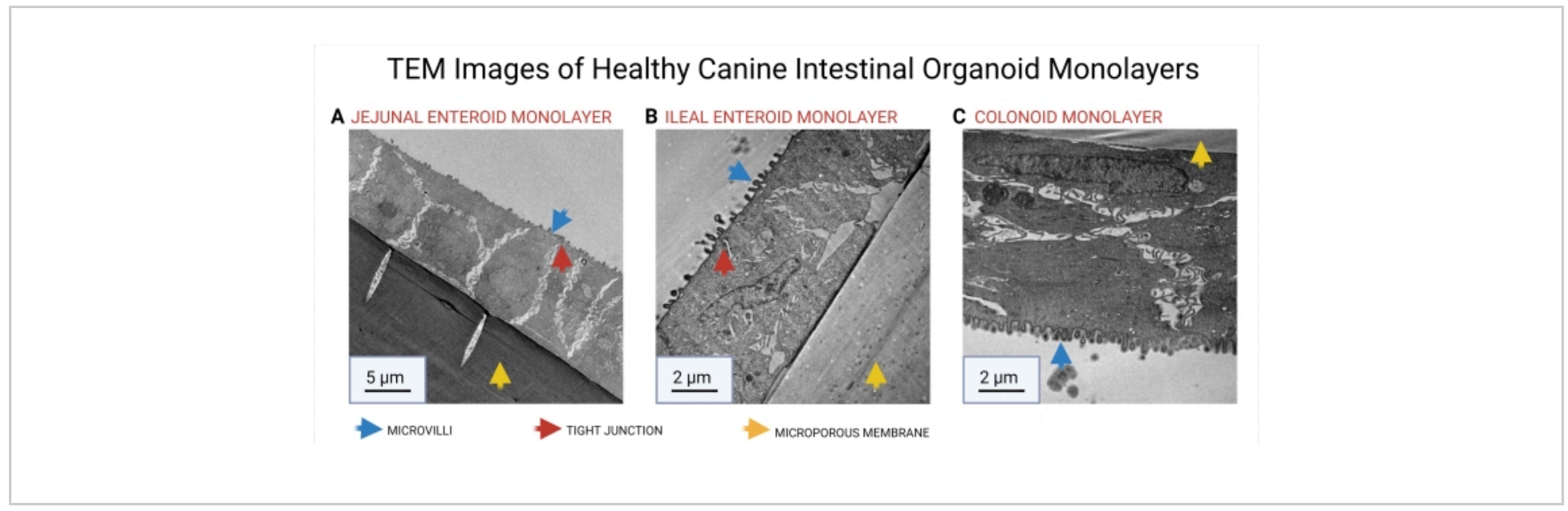

Figure 9: TEM images of healthy canine intestinal organoid monolayers. TEM was used to reveal the cellular microarchitecture of jejunal (A), ileal (B), and colonic (C) organoid monolayers. The microporous membrane of the permeable support insert is marked with a yellow arrow. The presence of microvilli (blue arrow) and tight junctions (red arrow $)$ is delineated in the images. Abbreviation: TEM $=$ Transmission electron microscopy. Scale bars $=5 \mu \mathrm{m}(\mathbf{A}), 2 \mu \mathrm{m}(\mathbf{B}$, C). Please click here to view a larger version of this figure.

Both TEER measurements and light microscopy should be performed to ensure that the system is ready for permeability testing. The assay is ready for use when TEER reaches a steady state, while light microscopy will help to exclude damage or overgrowth of the organoids. A summary of typical TEER measurements of five groups consisting of canine jejunal enteroids and colonoids is presented in Figure 6.

Table 1: Composition of organoid media and FAA. The complete composition of $\mathrm{CMGF}+\mathrm{CMGF}+\mathrm{R} / \mathrm{G}$, and $\mathrm{FAA}$ are summarized in this table. Abbreviations: ROCK $=$ rhoassociated kinase; GSKi $\beta$ = glycogen synthase kinase beta; $\mathrm{CMGF}+\mathrm{R} / \mathrm{G}=$ Complete medium with growth factors enhanced with ROCK inhibitor and GSKiß; CMGF+ = Complete medium with growth factors but without ROCK inhibitor or GSKiß; FAA = formalin-acetic acid-alcohol; EGF =epidermal growth factor. This table is adapted from ${ }^{38}$. Please click here to download this Table.

Supplemental Table 1: Table for note-taking on canine organoid the permeable support system. Abbreviations: TEER = transepithelial electrical resistance; $\mathrm{ROCK}=$ rhoassociated kinase; GSKi $\beta$ = glycogen synthase kinase beta; $\mathrm{CMGF}+\mathrm{R} / \mathrm{G}=$ Complete medium with growth factors enhanced with ROCK inhibitor and GSKiß; CMGF+ = Complete medium with growth factors but without ROCK inhibitor or GSKiß. Please click here to download this Table. 


\section{Discussion}

Canine intestinal organoid cultures in the permeable support apparatus are a unique concept connecting traditional drug permeability assays ${ }^{40}$ with a novel in vitro canine model ${ }^{41}$. Different types of canine intestinal organoids can be used and evaluated based on the goal of the experiment with minimal adjustments. Testing multiple concentrations of the drug of interest in 3-4 wells per group is recommended. The concentrations may be based on the expected intestinal concentration of the drug. Furthermore, using previous research may help determine appropriate time points for the study design. Appropriate documentation of study design should be made to increase replicability and assist in troubleshooting.

This technology does have several limitations due to the novelty of the method ${ }^{42}$, mostly due to the lack of standardization in experimental design and execution of the protocol across laboratories. This lack of standardization has been acknowledged by other groups ${ }^{43}$, and the Canine 3D Organoid Monolayer Protocols will lead to interlaboratory reproducibility and introduce standardization to this system. Standardized approaches to data analysis improve replicability and can strengthen results of preliminary drug testing using the canine organoids in the permeable supports system across different laboratories. The canine 3D organoid model also lacks data sets comparing in vitro $\mathrm{P}_{\mathrm{app}}$ values of model drugs to their known human or canine in vivo intestinal absorption, much like Caco- 2 cells ${ }^{44,45,46}$. Once such data has been generated, this canine organoid model may be utilized to assess intestinal permeability during drug development.

It is crucial that care is taken when seeding the organoids on the permeable support system to seed a high enough density of appropriately dissociated cells. The TEER values of the system are more reliable and reproducible when grown in strict monolayers. Prolonged culture of the monolayers can lead to an exponential increase in TEER values reaching further from physiological values of the intestine. H\&E sections of such 3D structures then show several layers of cells above each other with altered structures of enterocytes closer to the membrane.

After successful expansion of the canine intestinal organoid monolayer, results can be analyzed the same way as traditional $2 \mathrm{D}$ cell assays by calculating a drug's apparent permeability coefficient $\left(\mathrm{P}_{\mathrm{app}}\right)$ formula ${ }^{44}$. The $\mathrm{P}_{\text {app value }}$ (see Eq (2)) describes the rate of transport across the cellular monolayer $^{47}$.

$P_{a p p}=\frac{d Q}{d t} \times \frac{1}{A \times C_{0}}(\mathbf{2})$

The $\frac{d Q}{d t}$ is the initial slope of the concentration versus the time curve (e.g., nmol/s). $A$ is the area of the insert $\left(\mathrm{cm}^{2}\right)$, and $C_{0}$ is the initial concentration of the drug or compound in the donor chamber ${ }^{37}$. Reliable recognition of monolayer integrity is a crucial part of the permeability assay requiring standardization. Light microscopy and TEER measurements are recommended to assess canine organoids in a permeable support system and help determine the correct timing of the experiment. Additionally, zero molecular permeability markers (e.g., FITC-dextran, Lucifer yellow, PEG-400) can be used to functionally evaluate organoid monolayer integrity. Attention must be paid if the compound tested is affected by a transporter. P-glycoprotein (P-gp) is used as a common efflux pump example. An efflux ratio must be generated (Papp,BLAP/Papp,AP-BL) with comparison to a well-known P-gp probe substrate. 
Light microscopy (plain or enhanced with phase contrast) is an invaluable method to check for the integrity of the $2 D$ or $3 D$ monolayer and the filter insert while assessing possible cellular overgrowth. Figure 7 can serve as a guide to recognizing healthy canine intestinal organoid cell cultures. TEER values are an important measure of the formation of intercellular junctions and differentiation of the organoid cultures into an intact intestinal epithelium. The canine intestinal organoids differentiate into enterocytes and goblet cells (Figure 6). These mucus-producing cells allow for the study of drug-mucus interactions, which has been difficult to achieve using traditional $2 \mathrm{D}$ cell cultures ${ }^{48}$. The presence of enteroendocrine cells has been previously confirmed in canine intestinal organoids by Chandra et al. ${ }^{33}$.

Further characterization of the canine organoid monolayers derived from jejunal, ileal, and colonic organoids using TEM is provided. TEM images show cellular microarchitecture, including tight junctions and microvilli formation, further illustrating the complexity and usefulness of these organoid models in translational medicine. Based on the experimental results, the organoid cultures on the permeable support were ready for experimentation between days 11 and day 13 post seeding (Figure 9). The TEER values at this time point ranged between 1,500 and 2,500 $\Omega . \mathrm{cm}^{2}$. The plateau phase of TEER values lasts for a very limited window of time where the experiment must be started before the TEER values begin to decline slowly. TEER values can also be a crucial part of displaying important experimental results as some drugs or drug product excipients may interact with the monolayer (e.g., tight junctions), which can greatly impact TEER value readouts. This alone can serve as data for an experiment.

Canine intestinal organoids in a dual-chamber cell culture apparatus can be applied in fields other than oral drug permeability due to the unique architecture of the resultant cell monolayer. For example, they can be used in microbiology research (e.g., the impact of altering GI microbial flora), viral uptake studies, drug-drug interactions, and drug transport mechanisms ${ }^{49}$. The donor chamber is typically filled with the test drug or compound of choice, and aliquots from the receiver chamber are taken at various time points. These aliquots can be analyzed using high-performance liquid chromatography, mass spectrometry, enzyme-linked immunosorbent assay, or other techniques to determine the amount and speed by which the solute permeates through the monolayer.

These studies require an intact monolayer to assess drug permeability accurately. This typically requires growing monolayers in excess of those needed to account for unusable wells. Organoid cell monolayers can also be used to measure viral uptake either from the apical or basal side of a monolayer, with readouts including immunofluorescence assays-utilizing antibodies to detect the cellular uptake of the virus. Finally, multiple drugs (i.e., substrate and inhibitor) can be applied to the donor chamber to identify transporter-based drug-drug interactions.

Based on the current observations, these methods will not only be applicable to canine organoids in culture inserts but will also be suitable for other veterinary species and organ systems, with minor modifications needed to best suit the species or organ model of choice. Protocols for canine intestinal organoid growth had to be adjusted based on the unique properties of the culture. Thus, the protocol can be adjusted to another species but will require subtle changes to the protocol. Modifications may begin with changes to cell seeding density and expand to changes in the media composition to properly differentiate the organoids of interest. 
The standardization, detailed documentation of experimental procedures, and consistent monitoring of the cell monolayers are crucial practices needed across the permeable support assays and are not limited to the canine system. These possible species or organ modifications are critical to document and report for further advances in the field. This model has several limitations, for example, its cost requirements, interlaboratory variability, and limited data on the ability to predict intestinal absorption in vivo. Dogs, in some cases, possess different drug transporters and metabolizing enzymes than humans ${ }^{50}$.

Further, the canine organoid system must be tested on a variety of other dual-chamber apparatuses from other manufacturers to determine the suitability of such a model (e.g., the suitability of different filter membrane compositions must be determined). Another disadvantage is that the drug permeability experiment part of the manuscript is less descriptive than the previous parts. This is caused by an excess of information in this field. The goal for this part of the manuscript was to describe these methods in a modifiable way while not cutting the edges of cornerstones of these experiments. More detailed information on permeability experiments has been collected by Hubatsch et al. ${ }^{37}$. Additionally, permeable inserts can be used in coculture, cell migration, and invasion assay experiments ${ }^{4}$.

In conclusion, the canine intestinal organoids in dual-chamber culture apparatuses have the potential to be used in a wide range of applications, including biomedical fields and translational medicine, to name a few. The protocols create several strategies to plan an experiment and promote interlaboratory data dependability for organoid models across the field of biology.

\section{Disclosures}

K. Allenspach is a co-founder of LifEngine Animal Health and $3 \mathrm{D}$ Health Solutions. She serves as a consultant for Ceva Animal Health, Bioiberica, LifeDiagnostics, Antech Diagnostics, Deerland Probiotics, and Mars. J.P. Mochel is a co-founder of LifEngine Animal Health and 3D Health Solutions and serves as a consultant for Ceva Animal Health and Ethos Animal Health. This article reflects the views of the authors and should not be construed to represent Food and Drug Administration's endorsement, view, or policies. Other authors do not have any conflict of interest to declare.

\section{Acknowledgments}

We want to express gratitude to the Veterinary Diagnostic Laboratory of lowa State University employees, namely Haley Lambert, Emily Rahe, Rosalyn Branaman, Victoria Green, and Jennifer Groeltz-Thrush, for the timely processing of samples. We would also like to thank Jodi Smith and Bethann Valentine for providing material for the permeability experiments. We also want to thank David Diaz-Reganon for his help with Figure 9. Except for Figure 6, all figures were created in BioRender.com. The authors wish to acknowledge support from the Faculty Startup, ISU VPR Miller Award, ISU VPR Miller Award, and NSF SBIR subaward to ISU \# 1912948

\section{References}

1. Ghaffarian, R., Muro, S. Models and methods to evaluate transport of drug delivery systems across cellular barriers. Journal of Visualized Experiments : JoVE. 80 (80), 50638 (2013).

2. Youhanna, S., Lauschke, V. M. The Past, present and future of intestinal in vitro cell systems for drug absorption 
studies. Journal of Pharmaceutical Sciences. 110 (1), 50-65 (2021).

3. Belic, S. et al. Comparative analysis of inflammatory cytokine release and alveolar epithelial barrier invasion in a transwell囚bilayer model of mucormycosis. Frontiers in Microbiology. 9, 3204 (2019).

4. Justus, C. R., Leffler, N., Ruiz-Echevarria, M., Yang, L. V. In vitro cell migration and invasion assays. Journal of Visualized Experiments: JoVE. (88), 51046 (2014).

5. Rönkkö, S., Vellonen, K. S., Järvinen, K., Toropainen, E., Urtti, A. Human corneal cell culture models for drug toxicity studies. Drug Delivery and Translational Research. 6 (6), 660-675 (2016).

6. Dahlgren, D., Lennernäs, H. Intestinal permeability and drug absorption: predictive experimental, computational and in vivo approaches. Pharmaceutics. 11 (8), 411 (2019).

7. Schoultz, I., Keita, Å. V. The intestinal barrier and current techniques for the assessment of gut permeability. Cells. 9 (8), 1909 (2020).

8. Hilgers, A. R., Conradi, R. A., Burton, P. S. Caco-2 cell monolayers as a model for drug transport across the intestinal mucosa. Pharmaceutical Research. 7 (9), 902-910 (1990).

9. Natoli, M., Leoni, B. D., D’Agnano, I., Zucco, F., Felsani, A. Good Caco-2 cell culture practices. Toxicology in Vitro. 26 (8), 1243-1246 (2012).

10. Kapałczyńska, M. et al. $2 \mathrm{D}$ and $3 \mathrm{D}$ cell cultures - a comparison of different types of cancer cell cultures. Archives of Medical Science. 14 (4), 910-919 (2018).

11. Sun, H., Chow, E. C. Y., Liu, S., Du, Y., Pang, K. S. The Caco-2 cell monolayer: Usefulness and limitations.
Expert Opinion on Drug Metabolism and Toxicology. 4 (4), 395-411 (2008).

12. Chandler, M. et al. Obesity and associated comorbidities in people and companion animals: a One Health perspective. Journal of Comparative Pathology. 156 (4), 296-309 (2017).

13. Coelho, L. P. et al. Similarity of the dog and human gut microbiomes in gene content and response to diet. Microbiome. 6 (1), 72 (2018).

14. Galeta, P., Lázničková-Galetová, M., Sablin, M., Germonpré, M. Morphological evidence for early dog domestication in the European Pleistocene: New evidence from a randomization approach to group differences. Anatomical Record. 304 (1), 42-62 (2021).

15. Kleinert, M. et al. Animal models of obesity and diabetes mellitus. Nature Reviews Endocrinology. 14 (3), 140-162 (2018).

16. Allenspach, K., Wieland, B., Gröne, A., Gaschen, F. Chronic enteropathies in dogs: Evaluation of risk factors for negative outcome. Journal of Veterinary Internal Medicine. 21 (4), 700-708 (2007).

17. Wang, J. et al. Proliferative and invasive colorectal tumors in pet dogs provide unique insights into human colorectal cancer. Cancers. 10 (9), 330 (2018).

18. Gillespie, V., Baer, K., Farrelly, J., Craft, D., Luong, R. Canine gastrointestinal stromal tumors: Immunohistochemical expression of CD34 and examination of prognostic indicators including proliferation markers Ki67 and AgNOR. Veterinary Pathology. 48 (1), 283-291 (2011). 
19. Chandra, L. et al. Derivation of adult canine intestinal organoids for translational research in gastroenterology. BMC Biology. 17 (1), 33 (2019).

20. Schneider, B. et al. Model-based reverse translation between veterinary and human medicine: the One Health initiative. CPT: Pharmacometrics and Systems Pharmacology. 7 (2), 65-68 (2018).

21. Artursson, P., Palm, K., Luthman, K. Caco-2 monolayers in experimental and theoretical predictions of drug transport. Advanced Drug Delivery Reviews. 22 (1-2), 67-84 (1996).

22. Balimane, P. V., Han, Y.H., Chong, S. Current industrial practices of assessing permeability and P-glycoprotein interaction. AAPS Journal. 8 (1), E1-13 (2006).

23. Sambuy, Y. et al. The Caco-2 cell line as a model of the intestinal barrier: Influence of cell and culture-related factors on Caco-2 cell functional characteristics. Cell Biology and Toxicology. 21 (1), 1-26 (2005).

24. Calcagno, A. M., Ludwig, J. A., Fostel, J. M., Gottesman, M. M., Ambudkar, S. V. Comparison of drug transporter levels in normal colon, colon cancer, and caco-2 cells: Impact on drug disposition and discovery. Molecular Pharmaceutics. 3 (1), 87-93 (2006).

25. Hilgendorf, C. et al. Expression of thirty-six drug transporter genes in human intestine, liver, kidney, and organotypic cell lines. Drug Metabolism and Disposition. 35 (8), 1333 LP-1340 (2007).

26. Seithel, A., Karlsson, J., Hilgendorf, C., Björquist, A., Ungell, A. L. Variability in mRNA expression of ABC- and SLC-transporters in human intestinal cells: Comparison between human segments and Caco-2 cells. European
Journal of Pharmaceutical Sciences. 28 (4), 291-299 (2006).

27. Volpe, D. A. Transporter assays as useful in vitro tools in drug discovery and development. Expert Opinion on Drug Discovery. 11 (1), 91-103 (2016).

28. Hoffmann, P. et al. Caco-2/HT29-MTX co-cultured cells as a model for studying physiological properties and toxin-induced effects on intestinal cells. PLOS ONE. 16 (10), e0257824 (2021).

29. Sun, H., Chow, E. C. Y., Liu, S., Du, Y., Pang, K. S. The Caco-2 cell monolayer: Usefulness and limitations. Expert Opinion on Drug Metabolism and Toxicology. 4 (4), 395-411 (2008).

30. Thummel, K. E. et al. Transcriptional control of intestinal cytochrome P-4503A by 1a,25-dihydroxy vitamin D3. Molecular Pharmacology. 60 (6), 1399-1406 (2001).

31. Kodama, N. et al. Characteristic analysis of intestinal transport in enterocyte-like cells differentiated from human induced pluripotent stem cells. Drug Metabolism and Disposition. 44 (10), 1662-1667 (2016).

32. Akazawa, T. et al. Application of intestinal epithelial cells differentiated from human induced pluripotent stem cells for studies of prodrug hydrolysis and drug absorption in the small intestine. Drug Metabolism and Disposition: The Biological Fate of Chemicals. 46 (11), 1497-1506 (2018).

33. Lo, B., Parham, L. Ethical issues in stem cell research. Endocrine Reviews. 30 (3), 204-213 (2009).

34. Ambrosini, Y. M. et al. Recapitulation of the accessible interface of biopsy-derived canine intestinal organoids to study epithelial-luminal interactions. PLoS ONE. 15 (4), e0231423 (2020) 
35. Zdyrski, C. et al. Su124 homology directed repair in canine duodenal enteroids to mimic the wild-type Pglycoprotein mutation. Gastroenterology. 160 (6), S-625S-626 (2021).

36. Nantasanti, S. et al. Disease modeling and gene therapy of copper storage disease in canine hepatic organoids. Stem Cell Reports. 5 (5), 895-907 (2015).

37. Hubatsch, I., Ragnarsson, E. G. E., Artursson, P. Determination of drug permeability and prediction of drug absorption in Caco-2 monolayers. Nature Protocols. 2 (9), 2111-2119 (2007).

38. Gabriel, V. et al. Standardization and maintenance of 3D canine hepatic and intestinal organoid cultures for use in biomedical research. Journal of Visualized Experiments: JoVE. (179), e63515 (2022).

39. Frost, T. S., Jiang, L., Lynch, R. M., Zohar, Y. Permeability of epithelial/endothelial barriers in transwells and microfluidic bilayer devices. Micromachines. 10 (8), 533 (2019).

40. van Breemen, R. B., Li, Y. Caco-2 cell permeability assays to measure drug absorption. Expert Opinion on Drug Metabolism and Toxicology. 1 (2), 175-185 (2005).

41. Huch, M., Knoblich, J. A., Lutolf, M. P., MartinezArias, A. The hope and the hype of organoid research. Development. 144 (6), 938-941 (2017).

42. Sato, T. et al. Single Lgr5 stem cells build crypt-villus structures in vitro without a mesenchymal niche. Nature. 459 (7244), 262-265 (2009).

43. Olivatti, T. O. F., Alcantara, G. P., Lemos, A. C. C. E., Silva, M. G. da, Miot, H. A. Standardization of organoid culture for evaluation of melanogenesis induced by UVB,
UVA and visible light. Anais Brasileiros de Dermatologia. 95 (1), 46-51 (2020).

44. Volpe, D. A. et al. Classification of drug permeability with a Caco-2 cell monolayer assay. Clinical Research and Regulatory Affairs. 24 (1), 39-47 (2007).

45. Chen, C., Ma, M. G., Fullenwider, C. L., Chen, W. G., Sadeque, A. J. M. Biopharmaceutics permeability classification of lorcaserin, a selective 5-hydroxytryptamine 2C agonist: Method suitability and permeability class membership. Molecular Pharmaceutics. 10 (12), 4739-4745 (2013).

46. Jarc, T. et al. Demonstrating suitability of the Caco-2 cell model for BCS-based biowaiver according to the recent FDA and ICH harmonised guidelines. Journal of Pharmacy and Pharmacology. 71 (8), 1231-1242 (2019).

47. Newby, D., Freitas, A. A., Ghafourian, T. Decision trees to characterise the roles of permeability and solubility on the prediction of oral absorption. European Journal of Medicinal Chemistry. 90, 751-765 (2015).

48. Navabi, N., McGuckin, M. A., Lindén, S. K. Gastrointestinal cell lines form polarized epithelia with an adherent mucus layer when cultured in semi-wet interfaces with mechanical stimulation. PLOS ONE. 8 (7), e68761 (2013).

49. Puschhof, J. et al. Intestinal organoid cocultures with microbes. Nature Protocols. 16 (10), 4633-4649 (2021).

50. Martinez, M. N., Mochel, J. P., Neuhoff, S., Pade, D. Comparison of canine and human physiological factors: understanding interspecies differences that impact drug pharmacokinetics. The AAPS Journal. 23 (3), 59 (2021). 